
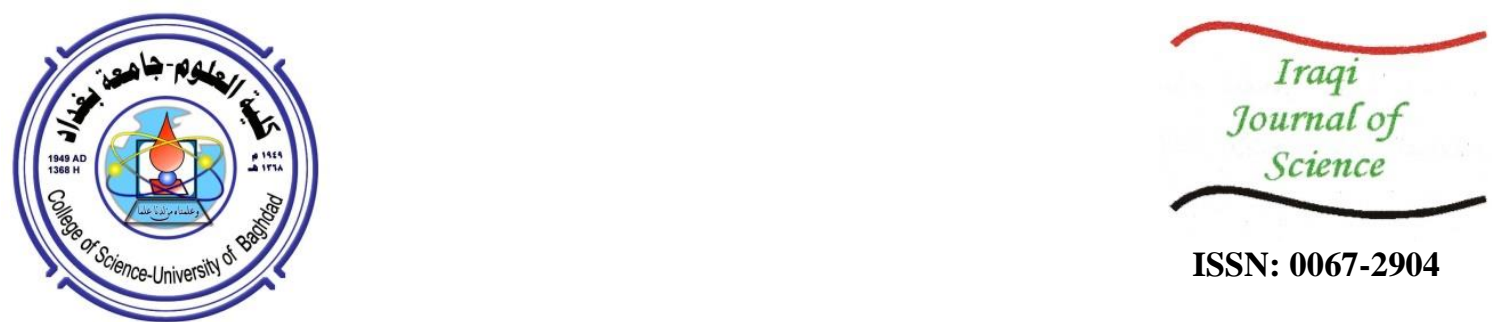

ISSN: 0067-2904

\title{
On the Characteristic Properties for Subclasses of the Higher Derivative of Analytic Functions
}

\author{
Kassim A. Jassim*', Zainab H. Mahmood ${ }^{2}$, Buthyna N.Shihab ${ }^{3}$ \\ ${ }^{1}$ Department of Mathematics, College of Science, University of Baghdad, Baghdad, Iraq \\ ${ }^{2}$ Department of physical, College of Science, University of Baghdad, Baghdad, Iraq \\ ${ }^{3}$ Department of Mathematics, College of Education Abn-Alhaithem for pure Sciences, University of Baghdad, \\ Baghdad, Iraq
}

\begin{abstract}
In the present paper, we introduce two subclasses, $S^{*} C(\rho, \sigma, \gamma, s, d)$ and $T S^{*} C(\rho, \sigma, \gamma, s, d)$, of analytic functions . Coefficients bounds for these subclasses are calculated.

The main purpose of this article is to originate characteristic properties of the functions in the above subclasses.
\end{abstract}

Keywords Multivalent function, Analytic function, Starlike function, Convex function.

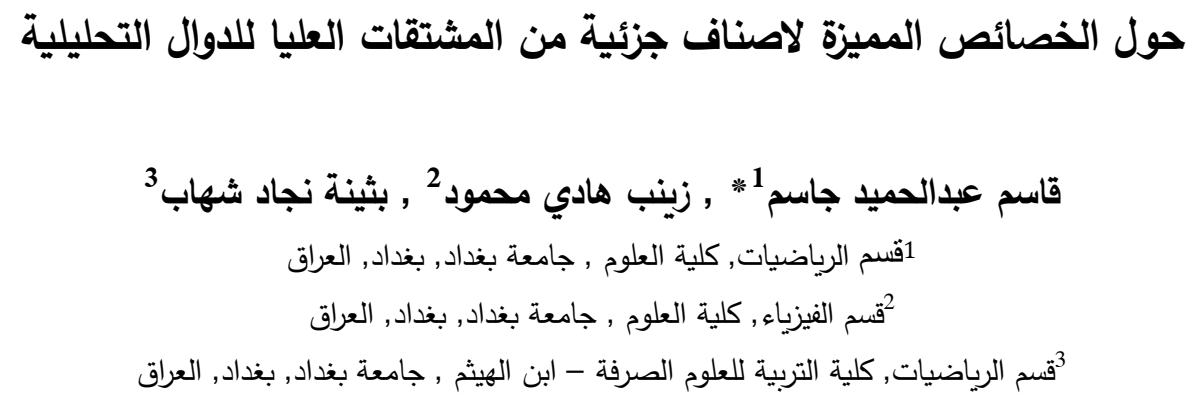

\section{Introduction}

Let $\mathcal{H}(s)$ denotes the class of analytic functions $f(z)$ in the open unit disk $U=\{z \in C:|z|<1\}$, of the form

$$
f(z)=z^{s}+\sum_{r=s+1}^{\infty} a_{n} z^{r}
$$

Moreover, we will denote by $S(\mathrm{~s})$ the family of all functions in $\mathcal{H}(s)$ which are univalent in $U$. Let $\tau(s)$ denotes the subclass of all functions $f(z)$ involving non positive coefficients, in $\mathcal{H}(s)$ of the form

$$
f(z)=z^{s}-\sum_{r=s+1}^{\infty} a_{n} z^{r}
$$

Many authors [1-7] studied classes like these and described many properties for analytic functions.

Prajapat [8] presented the subclasses $R_{a}^{\square}(r, \rho), V_{a}^{\square}(r, \rho), T_{a}^{\square}(r, \sigma, \rho)$ and $T_{a}^{\square}(r, \sigma, \rho)$ of the class of analytic functions $f(z)$ in the open unit disk $U \mathcal{H}(1)$ and a few incorporation connections were set up for the above subclasses. Likewise, Prajapat [9] presented an intriguing subclass $\chi_{t}(\gamma)$ of analytic and close to convex functions in $U$. 
Also, Mustafa [10] presented the subclass $K(\rho, \sigma), \rho, \sigma \in[0,1)$, which is the speculation of the near curved capacities, called near arched, regarding a starlike capacity $\mathrm{g}(\mathrm{z})$ of order $\rho(\rho \in[0,1))$ and type $\sigma(\sigma \in[0,1))$ of analytic functions .

In $[10,11]$, Mustafa discovered adequate conditions for the boundaries of the standardized Wright capacities to be in the class $K(\rho, \sigma)$.

As of late, Panigrahi and Murugusundaramoorthy [12] presented another subclass which is denoted by $M_{\lambda, \delta}^{k, t}(\rho)$. They found sharp gauges for the qualification of the coefficients of the limits having a spot with this class.

As it will in general be seen from the recently referenced assessments, a segment of the noteworthy and all around investigated subclasses of $\mathrm{S}$ are the classes $S^{*}(\rho)$ and $C(\rho)$ defined as follows.

Definition 1.1. [13-15]. The class of starlike functions $S^{*}(\rho)$ of order $\rho(\rho \in[0,1))$ and the class of convex functions $C(\rho)$ of order $\rho(\rho \in[0,1))$ are defined, respectively, by

and

$$
S^{*}(\rho)=\left\{f \in \mathcal{H}(1): \operatorname{Re}\left(\frac{z f^{\prime}(z)}{f(z)}\right)>\rho, z \in U\right\}, \rho \in[0,1)
$$

$$
C(\rho)=\left\{f \in \mathcal{H}(1): \operatorname{Re}\left(1+\frac{z f^{\prime \prime}(z)}{f^{\prime}(z)}\right)>\rho, z \in U\right\}, \rho \in[0,1)
$$

Moreover, we will denote

$$
\tau S^{*}(\rho, \sigma)=S^{*}(\rho, \sigma) \cap \tau \text { and } \tau C(\rho, \sigma)=C(\rho, \sigma) \cap \tau \text {, where } \tau(1)=\tau \text {. }
$$

Therefore,the generalization classes $S^{*}(\rho)$ and $C(\rho)$ are denoted, respectively, as $S^{*}(\rho, \sigma)$ and $C(\rho, \sigma)$ and defined by

and

$$
S^{*}(\rho, \sigma)=\left\{f \in \mathcal{H}(1): \operatorname{Re}\left(\frac{z f^{\prime}(z)}{\sigma z f^{\prime}(z)+(1-\sigma) f(z)}\right)>\rho, z \in U\right\}, \rho, \sigma \in[0,1)
$$

$$
C(\rho, \sigma)=\left\{f \in \mathcal{H}(1): \operatorname{Re}\left(\frac{f^{\prime}(z)+z f^{\prime \prime}(z)}{f^{\prime}(z)+\sigma z f^{\prime \prime}(z)}\right)>\rho, z \in U\right\}, \rho, \sigma \in[0,1)
$$

The classes $\tau S^{*}(\rho, \sigma)$ and $\tau C(\rho, \sigma)$ were studied by Altıntaş and Owa [15] . Likewise, certain conditions for hypergeometric works and summed up Bessel capacities for these classes were emphasized by Altintas and Owa [16] , Moustafa [17] , and Porwal and Dixit [18].

Therefore, based on the above mentioned studies, we define a generalized unification of the function classes $S^{*}(\rho, \sigma)$ and $C(\rho, \sigma)$, as follows.

Definition 1.2. A function $f \in \mathcal{H}(s)$ given by (1) is said to be in the subclass $S^{*} C(\rho, \sigma, \gamma, s, d)(\rho, \sigma \in$ $[0,1), \gamma \in[0,1])$ on the off chance that the accompanying condition is fulfilled.

$$
\operatorname{Re}\left\{\frac{z^{d+1} f^{d+1}(z)+\gamma z^{d+2} f^{d+2}(z)}{\gamma^{d+1}\left(f^{d+1}(z)+\sigma z f^{d+2}(z)\right)+(1-\gamma)\left(\sigma z f^{d+1}(z)+(1-\sigma) z^{d} f^{d}(z)\right.}\right\}>\rho \quad z \in U
$$

Also, we will use

$\tau(s) S^{*} C(\rho, \sigma, \gamma, s, d)=S^{*} C(\rho, \sigma, \gamma, s, d) \cap \tau(s)$.

We note the following:

$S^{*} C(\rho, \sigma, 0,1,0)=S^{*}(\rho, \sigma)$ studied by Mustafa [11];

$S^{*} C(\rho, \sigma, 1,1,0)=C(\rho, \sigma)$ studied by Mustafa [11];

$S^{*} C(\rho, 0,0,1,0)=S^{*}(\rho)$ studied by Siverman [19];

$S^{*} C(\rho, 0,1,1,0)=C(\rho)$ studied by Siverman [19];

$\tau S^{*} C(\rho, \sigma, 0,1,0)=\tau S^{*}(\rho, \sigma)$ studied by Altintas, Altintas and Irmak, altintas and Ozkan and Porwal [20-24];

$\tau S^{*} C(\rho, \sigma, 1,1,0)=\tau C(\rho, \sigma)$ studied by Altintas and Owa[16]; 
$\tau S^{*} C(\alpha, 0,0,1,0)=\tau S^{*}(\rho)$ studied by Siverman [19];

$\tau S^{*} C(\alpha, 0,1,1,0)=\tau C(\rho)$ studied by Siverman [19].

The main purpose of this paper is to satisfy characteristic properties of the subclasses

$S^{*} C(\rho, \sigma, \gamma, s, d)$ and $\tau(p) S^{*} C(\rho, \sigma, \gamma, s, d)$

( $\rho, \sigma \in[0,1), \gamma \in[0,1]$ ) of analytic functions $U$. Hence, coefficient bounds are also given for the functions belonging to the above subclasses.

A sufficient condition for functions in $S^{*} C(\rho, \sigma, \gamma, s, d), \rho, \sigma \in[0,1), \gamma \in[0,1]$ is given by the following theorem.

Theorem 2.1. Let $f \in \mathcal{H}(s)$. Then, the capacity $f(\mathrm{z})$ has a place with the subclass $S^{*} C(\rho, \sigma, \gamma, s, d)$ where $(\rho, \sigma \in[0,1), \gamma \in[0,1])$ on the off chance that the accompanying condition is fulfilled:

$$
\begin{aligned}
& \sum_{r=s+1}^{\infty} \frac{r !}{(r-d) !}\{(r-d)(\gamma(1-\rho \sigma)(n-d-1)-\rho(\sigma(1-\gamma)+\gamma)+1)-\rho(1-\gamma)(1-\sigma)\}\left|a_{r}\right| \\
& \\
& \quad \leq(s-d)(1+\sigma(s-d-1))(\gamma(2-\rho)-1) \\
&+(1-\gamma)(2-\rho)(\sigma(s-d)+(1-\sigma)) \ldots \ldots(3)
\end{aligned}
$$

The result is sharp for the functions

$f_{r}(z)$

$$
+\frac{(s-d)(1+\sigma(s-d-1))(\gamma(2-\rho)-1)+(1-\gamma)(2-\rho)(\sigma(s-d)+(1-\sigma)) z^{r}}{\sum_{r=2}^{\infty} \frac{r !}{(r-d) !}\{(r-d)(\gamma(1-\rho \sigma)(r-d-1)-\rho(\sigma(1-\gamma)+\gamma)+1)-\rho(1-\gamma)(1-\sigma)\}}
$$
$z \in U, r=2,3, \ldots$

Proof . Let the inequality (3) holds true and $|z|=1$, then we have

It suffices to show that

$$
\left|\frac{z^{d+1} f^{d+1}(z)+\gamma z^{d+2} f^{d+2}(z)}{\gamma z^{d+1}\left(f^{d+1}(z)+\sigma z f^{d+2}(z)\right)+(1-\gamma)\left(\sigma z^{d+1} f^{d+1}(z)+(1-\sigma) z^{d} f^{d}(z)\right)}-1\right| \leq 1-\rho
$$

$$
\begin{aligned}
& \mid z^{d+1} f^{d+1}(z)+\gamma z^{d+2} f^{d+2}(z)-\gamma z^{d+1}\left(f^{d+1}(z)+\sigma z f^{d+2}(z)\right)-(1-\gamma) \sigma z^{d+1} f^{d+1}(z)+ \\
& \left((1-\sigma) z^{d} f^{d}(z)\right)|-(1-\rho) \quad| \gamma z^{d+1}\left(f^{d+1}(z)+\sigma z f^{d+2}(z)\right)+(1-\gamma)\left(\sigma z^{d+1} f^{d+1}(z)+(1-\right. \\
& \left.\sigma) z^{d} f^{d}(z)\right) \mid \\
& =\mid \frac{s !}{(s-d) !}\{((s-d)(1+\gamma(s-d-1))-\gamma(s-d)(1+\sigma(s-d-1))-(1-\gamma)(\sigma(s-d)+ \\
& (1-\sigma)))\} z^{s}+ \\
& \sum_{r=s+1}^{\infty} \frac{r !}{(r-d) !}((r-d)(1+\gamma(r-d-1))-\gamma(r-d)(1+\sigma(r-d-1))-(1 \\
& \quad-\gamma)(\sigma(r-d)+(1-\sigma))) a_{r} z^{r} \mid \\
& \quad-(1-\rho) \mid \frac{s !}{(s-d) !}\{\gamma(s-d)((1+\sigma(s-d-1)))+(1-\gamma)(\sigma(s-d)+(1-\sigma))\} z^{s} \\
& \quad+\sum_{r=s+1}^{\infty} \frac{r !}{(r-d) !}(\gamma(r-d)(1+\sigma(r-d-1))+(1-\gamma)(\sigma(r-d)+(1-\sigma))) a_{r} \mid \\
& \leq \sum_{r=s+1}^{\infty} \frac{r !}{(r-d) !}\{(r-d)(\gamma(1-\alpha \sigma)(r-d-1)-\rho(\sigma(1-\gamma)+\gamma)+1)-\rho(1-\gamma)(1-\sigma)\}- \\
& (s-d)(1+\sigma(s-d-1))(\gamma(2-\rho)-1)+(1-\gamma)(2-\rho)(\sigma(s-d)+(1-\sigma)) \leq 0,
\end{aligned}
$$

by hypothesis.

By setting $s=1$ and $d=0$ in Theorem 2.1, the accompanying results are obtained by Mustafa [11].

Corollary 2.1. The capacity $\mathrm{f}(\mathrm{z})$ characterized by (1) has a place with the subclass $, \sigma, \gamma, 1,0),(\rho, \sigma \in[0,1), \gamma \in[0,1])$ on the off chance that the accompanying condition is fulfilled: 


$$
\begin{gathered}
\sum_{r=2}^{\infty}\{(r)(\gamma(1-\rho \sigma)(r-1)-\rho(\sigma(1-\gamma)+\gamma)+1)-\rho(1-\gamma)(1-\sigma)\}\left|a_{r}\right| \\
\leq(\gamma(2-\rho)-1)+(1-\gamma)(2-\rho)
\end{gathered}
$$

By setting $\gamma=0$ and $\gamma=1$, we can easily get the following corollaries.

Corollary 2.2. Let $\mathrm{f}(\mathrm{z})$ characterized by (1) has a place with the class $S^{*}(\rho, \sigma)(\rho, \sigma \in[0,1))$ if the following condition is

Sharpness is fulfilled for the capacities

$$
\sum_{r=2}^{\infty}\{r(1-\rho \sigma)-\rho(1-\sigma)\}\left|a_{r}\right| \leq(1-\rho)
$$

$$
f_{r}(z)=z+\frac{(1-\rho)}{r(1-\rho \sigma)-\rho(1-\sigma)} z^{r}, z \in U, r=2,3, \ldots
$$

Corollary 2.3. Let $\mathrm{f}(\mathrm{z})$ characterized by $(1)$ has a place with the class $\mathrm{C}(\rho, \sigma)(\rho, \sigma \in[0,1))$ if the following condition

$$
\sum_{r=2}^{\infty}\left\{r \left((1-(\rho \sigma)(r-1)-(\rho+1)\}\left|a_{r}\right| \leq(1-\rho)\right.\right.
$$

Sharpness is fulfilled for the capacities

$$
f_{r}(z)=z+\frac{(1-\rho)}{r((1-(\rho \sigma)(r-1)-\rho+1)} z^{r}, z \in U, r=2,3, \ldots
$$

By taking $\sigma=0$ in Corollaries 2.2 and 2.3, separately, we have the accompanying outcomes that are emphasized by Siverman [19].

Corollary 2.4. Let $f(\mathrm{z})$ characterized by $(1)$ has a place with the class $S^{*}(\rho)(\rho \in[0,1))$ on the off chance that the accompanying condition is fulfilled:

Sharpness is fulfilled for the capacities

$$
\sum_{r=2}^{\infty}(r-\rho)\left|a_{r}\right| \leq 1-\rho
$$

$$
f_{r}(z)=z+\frac{(1-\rho)}{(r-\rho)} z^{r} \quad z \in U, r=2,3, \ldots
$$

Corollary 2.5. Let $\mathrm{f}(\mathrm{z})$ characterized by $(1)$ has a place with the class $C(\rho)(\rho \in[0,1))$ on the off chance that the accompanying condition is fulfilled $\sum_{r=2}^{\infty} r(r-\rho)\left|a_{r}\right| \leq(1-\rho)$

Sharpness is fulfilled for the capacities

$$
f_{r}(z)=z+\frac{(1-\rho)}{r(r-\rho)} z^{r} \quad z \in U, r=2,3, \ldots
$$

Remark 2.1. Multiple results of the properties given by Corollaries 2.3 and 2.4 can be obtained for each of the classes studied by many researchers, especially including the various parameters.

For the function in the class $\tau(s) S^{*} C(\rho, \sigma, \gamma, s, d)$, the converse of Theorem 2.1 is also true.

Theorem 2.2. Let $f \in \tau(s)$. Then, the function $f(z)$ belongs to the class $S^{*} C(\rho, \sigma, \gamma, s, d)$, where $(\rho, \sigma \in[0,1), \gamma \in[0,1])$ if and only if

$$
\begin{aligned}
\sum_{r=s+1}^{\infty} \frac{r !}{(r-d) !}\{ & (r-d)(\gamma(1-\rho \sigma)(r-d-1)-\rho(\sigma(1-\gamma)+\gamma)+1)-\rho(1-\gamma)(1-\sigma)\} a_{r} \\
& \leq(s-d)(1+\sigma(s-d-1))(\gamma(2-\rho)-1) \\
& +(1-\gamma)(2-\rho)(\sigma(s-d)+(1-\sigma)) \ldots \ldots(3)
\end{aligned}
$$

Sharpness is fulfilled for the capacities

$$
\begin{aligned}
& f_{r}(z) \\
& =z \\
& -\frac{(s-d)(1+\sigma(s-d-1))(\gamma(2-\rho)-1)+(1-\gamma)(2-\rho)(\sigma(s-d)+(1-\sigma)) z^{r}}{\sum_{r=2}^{\infty} \frac{r !}{(r-d) !}\{(r-d)(\gamma(1-\rho \sigma)(r-d-1)-\rho(\sigma(1-\gamma)+\gamma)+1)-\rho(1-\gamma)(1-\sigma)\}}
\end{aligned}
$$


$z \in U, r=2,3, \ldots$

Proof. Let $f \in S^{*} C(\rho, \sigma ; \gamma), \rho, \sigma \in[0,1), \gamma \in[0,1]$. Then, according to Definition 1.2, we have

$\operatorname{Re}\left\{\frac{z^{d+1} f^{d+1}(z)+\gamma z^{d+2} f^{d+2}(z)}{\gamma z^{d+1}\left(f^{d+1}(z)+\sigma z f^{d+2}(z)\right)+(1-\gamma)\left(\sigma z^{d+1} f^{d+1}(z)+(1-\sigma) z^{d} f^{d}(z)\right)}\right\}>\rho, z \in U$

Also, we can easily show that the condition (5) holds true if

$\operatorname{Re}\left\{\frac{z^{d+1} f^{d+1}(z)+\gamma z^{d+2} f^{d+2}(z)}{\gamma z^{d+1}\left(f^{d+1}(z)+\sigma z f^{d+2}(z)\right)+(1-\gamma)\left(\sigma z^{d+1} f^{d+1}(z)+(1-\sigma) z^{d} f^{d}(z)\right)}-1\right\} \leq 1-\rho$

$\operatorname{Re}$

$\left\{\frac{\frac{s !}{(s-d) !}\{((s-d)(1+\gamma(s-d-1))-\gamma(s-d)(1+\sigma(s-d-1))-(1-\gamma)(\sigma(s-d)+(1-\sigma)))\} z^{s}+\sum_{r=2}^{\infty} \frac{r !}{(r-d) !}((r-d)(1+\gamma(r-d-1))-\gamma(r-d)(1+\sigma(r-d-1))-(1-\gamma)(\sigma(r-d)+(1-\sigma))) a_{r}}{\frac{s !}{(s-d) !}\{(r(s-d)((1+\sigma(s-d-1)))+(1-\gamma)(\sigma(s-d)+(1-\sigma)))\} z^{s}+\sum_{r=2}^{\infty} \frac{r(r-d) !}{(r)}(\gamma(r-d)(1+\sigma(r-d-1))+(1-\gamma)(\sigma(r-d)+(1-\sigma))) a_{n}}\right\} \leq$ $1-\rho$

The value of $z$ on the real axis so that $f(z)$ is real and letting $z \rightarrow 1^{-}$, we obtain

$$
\begin{aligned}
\sum_{r=s+1}^{\infty} \frac{r !}{(r-d) !}\{ & (r-d)(\gamma(1-s \sigma)(r-d-1)-\rho(\sigma(1-\gamma)+\gamma)+1)-\rho(1-\gamma)(1-\sigma)\} a_{r} \\
& \leq(s-d)(1+\sigma(s-d-1))(\gamma(2-\rho)-1) \\
& +(1-\gamma)(2-\rho)(\sigma(s-d)+(1-\sigma))
\end{aligned}
$$

By setting $s=1$ and $d=0$ in Theorem 2.2, the accompanying culminations are acquired by Mustafa [11].

Corollary 2.6. Let $\mathrm{f}(\mathrm{z})$ characterized by (2) has a place with the class $S^{*} C(\rho, \sigma, \gamma, 1,0)(\rho, \sigma$ $\in[0,1))$ on the off chance that the accompanying condition is fulfilled

$$
\begin{gathered}
\sum_{r=2}^{\infty}\{(r)(\gamma(1-\rho \sigma)(r-1)-\rho(\sigma(1-\gamma)+\gamma)+1)-\rho(1-\gamma)(1-\sigma)\} a_{r} \\
\leq(\gamma(2-\rho)-1)+(1-\gamma)(2-\rho)
\end{gathered}
$$

By setting $\gamma=0$ and $\gamma=1$, we can easily get the following corollaries.

Corollary 2.7. Let $\mathrm{f}(\mathrm{z})$ characterized by (2) has a place with the class $\tau S^{*}(\rho, \sigma)(\rho, \sigma \in[0,1))$ if the following condition is

$$
\sum_{r=2}^{\infty}\{(r)(1-\rho \sigma)-\rho(1-\sigma)\} a_{r} \leq(1-\rho)
$$

Sharpness is fulfilled for the capacities

$$
f_{r}(z)=z-\frac{(1-\rho)}{r(1-\rho \sigma)-\rho(1-\sigma)} z^{r}, z \in U, r=2,3, \ldots
$$

Corollary 2.8. Let $\mathrm{f}(\mathrm{z})$ characterized by (2) has a place with the class $\tau C(\rho, \sigma)(\rho, \sigma \in[0,1))$ on the off chance that the accompanying condition is fulfilled

$$
\sum_{r=2}^{\infty}\{r((1-\rho \sigma)(r-1)-\rho+1)\} a_{r} \leq(1-\rho)
$$

Sharpness is fulfilled for the capacities

$$
f_{r}(z)=z-\frac{(1-\rho)}{r((1-\rho \sigma)(r-1)-\rho+1)} z^{r}, z \in U, r=2,3, \ldots
$$

By taking $\sigma=0$ in Corollaries 2.7 and 2.8, individually, we have the accompanying outcomes.

Corollary 2.9. Let $f(\mathrm{z})$ characterized by (2) has a place with the class $\tau S^{*}(\rho)(\rho \in[0,1))$ on the off chance that the accompanying condition is fulfilled

Sharpness is fulfilled for the capacities

$$
\sum_{r=2}^{\infty}(r-\rho) a_{r} \leq(1-\rho)
$$

$$
f_{r}(z)=z-\frac{(1-\rho)}{(r-\rho)} \quad z \in U, r=2,3, \ldots
$$


Corollary 2.10. Let $f(\mathrm{z})$ characterized by (2) has a place with the class $\tau C(\rho)(\rho \in[0,1))$ on the off chance that the accompanying condition is fulfilled

Sharpness is fulfilled for the capacities

$$
\sum_{r=2}^{\infty} r(r-\rho) a_{r} \leq(1-\rho)
$$

$$
f_{r}(z)=z-\frac{(1-\rho)}{r(r-\rho)} \quad z \in U, r=2,3, \ldots
$$

Theorem 2.3. Let $f(\mathrm{z})$ characterized by (2) has a place with the class $\tau(s) S{ }^{*} C(\rho, \sigma, \gamma, s, d)$ $(\rho, \sigma \in[0,1), \gamma \in[0,1))$

Then

$$
\sum_{r=s+1}^{\infty}\left|a_{r}\right| \leq \frac{(s-d)(1+\sigma(s-d-1))(\gamma(2-\rho)-1)+(1-\gamma)(2-\rho)(\sigma(s-d)+(1-\sigma))}{\left.\frac{(s+1) !}{(s+1-d) !}(s+1-d)(\gamma(1-\rho \sigma)(s-d)-\rho(\sigma(1-\gamma)+\gamma)+1)-\rho(1-\gamma)(1-\sigma)\right\}}
$$

\section{Proof.}

Using Theorem 2.2, we write

$$
\begin{aligned}
& \quad \frac{(s+1) !}{(s+1-d) !}\{(s+1-d)(\gamma(1-\rho \sigma)(s-d)-\rho(\sigma(1-\gamma)+\gamma)+1)-\rho(1-\gamma)(1-\sigma)\} \sum_{r=2}^{\infty}\left|a_{r}\right| \\
& \leq \sum_{r=2}^{\infty} \frac{r !}{(r-d) !}\{(r-d)(\gamma(1-\rho \sigma)(r-d-1)-\rho(\sigma(1-\gamma)+\gamma)+1)-\rho(1-\gamma)(1-\sigma)\}\left|a_{r}\right| \\
& \leq(s-d)(1+\sigma(s-d-1))(\gamma(2-\rho)-1)+(1-\gamma)(2-\rho)(\sigma(s-d)+(1-\sigma)) .
\end{aligned}
$$

That is

$$
\sum_{r=s+1}^{\infty}\left|a_{r}\right| \leq \frac{(s-d)(1+\sigma(s-d-1))(\gamma(2-\rho)-1)+(1-\gamma)(2-\rho)(\sigma(s-d)+(1-\sigma))}{\frac{(s+1) !}{(s+1-d) !}\{(s+1-d)(\gamma(1-\rho \sigma)(s-d)-\rho(\sigma(1-\gamma)+\gamma)+1)-\rho(1-\gamma)(1-\sigma)\}}
$$

Thus, inequality ( 9 ) is provided.

By setting $s=1$ and $d=0$, the following corollaries are obtained by Mustafa [11].

Corollary 2.11. Let $f(\mathrm{z})$ characterized by (2) has a place with the class $\tau(s) S^{*} C(\rho, \sigma, \gamma, 1,0)$ $(\rho, \sigma \in[0,1), \gamma \in[0,1))$, we get

$$
\sum_{r=2}^{\infty}\left|a_{r}\right| \leq \frac{1-\rho}{\{2(\gamma(1-\rho \sigma)-\rho(\sigma(1-\gamma)+\gamma)+1)-\rho(1-\gamma)(1-\sigma)\}}
$$

Also, by setting $\gamma=0$ and $\gamma=1$ in Theorem 2.3, we show the accompanying outcomes separately emphasized by Mustafa [11].

Corollary 2.12. Let $f(z)$ characterized by (2) has a place with the class $\tau(1) S^{*}(\rho, \sigma)(\rho, \sigma \in[0,1))$. Then,

$$
\sum_{r=s+1}^{\infty}\left|a_{r}\right| \leq \frac{1-\rho}{2-(1+\sigma) \rho}
$$

Corollary 2.13. Let $f(\mathrm{z})$ characterized by (2) has a place with the class $\tau(1) C(\rho, \sigma)(\rho, \sigma$ $\in[0,1))$. Then,

$$
\sum_{r=s+1}^{\infty}\left|a_{r}\right| \leq \frac{1-\rho}{2(2-(1+\sigma) \rho)}
$$

Remark 2.4. Different aftereffects of the properties given by Corollaries 2.3 and 2.4 can be obtained for every one of the classes emohasized by numerous scientists, particularly including the different boundaries.

Numerous aftereffects of the coefficient gauges (given by Corollary 2.12 and Corollary 2.13) can in reality be found by altering the different boundaries included.

Moreover, by setting $\rho=\sigma=0$ in Corollaries 2.12 and 2.13, we obtain interesting results for the classes $\tau S^{*}$ and $\tau C$, respectively, obtained by Mustafa [11].

Corollary 2.14. Let $f(\mathrm{z})$ characterized by (2) has a place with the class $\tau S^{*}$. Then,

$$
\sum_{r=2}^{\infty}\left|a_{r}\right| \leq \frac{1}{2}
$$

Corollary 2.15. Let $f(z)$ characterized by (2) has a place with the $\operatorname{class} \tau C$. Then,

$$
\sum_{r=2}^{\infty}\left|a_{r}\right| \leq \frac{1}{4}
$$




\section{References}

1. Frasin, B. A. 2012. New subclasses of analytic functions: Journal of Inequalities and Applications, 24: $1-10$.

2. Cho, N. E. and Kim, J. A. 2006. Inclusion Properties of Certain Subclasses of Analytic Functions Defined by a Multiplier Transformation: Computers and Mathematics with Applications, 52: 323330.

3. Darus, M. and Faisal, I. 2012. Some subclasses of analytic functions of complex order defined by new differential operator: Tamkang Journal of Mathematics, 43: 223-242.

4. Gao, C. Y., Zhou, S. Q. 2005. On a class of analytic functions related to the class to the starlike functions: Kyungpook Mathematical Journal, 45: 123-130.

5. Kowalczyk, J., Les-Bomba, E. 2010. On a subclass of close-to-convex functions: Applied Mathematics Letters, 23: 1147-1151.

6. Xu, Q. H., Srivastava, H. M., Li, Z. 2011. A certain subclass of analytic and close-to-convex functions: Applied Mathematics Letters, 24: 396-401.

7. Wang, Z. G., Chen, D. Z. 2009. On a certain subclass of close-to-conex functions: Hacettepe Journal of Mathematics and Statistics, 38: 95-101.

8. Prajapat, J. K. 2012. Inclusion properties for certain class of analytic functions involving multiplier transformation operator: Journal of Classical Analysis, 1: 35-42.

9. Prajapat, J. K. 2016. A new subclass of close-to-convex functions: Surveys in Mathematics and Applications, 11: 11-19.

10. Mustafa, N. 2016. Close-toconvexity of normalized Wright functions: Dokuz Eylul University Faculty of Engineering Journal of Science and Engineering, 18: 290-303.

11. Mustafa, N. 2017. Characteristic properties of the new subclasses of analytic functions. Dokuz Eylul University Faculty of Engineering Journal of Science and Engineering, 19: 247-257

12. Panigrahi, T., Murugusundaramoorthy, G. 2016. On Successive Coefficient Estimate for Certain Subclass of Analytic Functions: Applied Mathematics ENotes, 16: 117-124.

13. Duren, P. L. 1983. Univalent Functions. Grundlehren der Mathematischen Wissenshaften, Bd. 259, New York, SpringerVerlag, 382p.

14. Goodman, A. W. 1983. Univalent Functions. Volume I, Washington, Polygonal, 246p.

15. Srivastava, H. M. and Owa, S. (Ed.) 1992. Current Topic in Analytic Function Theory. World Scientific Publishing Company, New Jersey, London, Hong Kong, 456p.

16. Altıntaş, O. and Owa, S. 1988. On subclasses of univalent functions with negative coefficients: Pusan Kyongnam Mathematical Journal, 4: 41-56.

17. Moustafa, A. O. 2009. A study on starlike and convex properties for hypergeometric functions: Journal of Inequalities in Pure and Applied Mathematics, 10: 1-16.

18. Porwal, S. and Dixit K. K. 2013. An application of generalized Bessel functions on certain analytic functions: Acta Universitatis Matthiae Belii series Mathematics, pp. 51-57.

19. Siverman, H. 1975. Univalent Functions with Negative Coefficients: American Mathematical Society, 51: 106-116.

20. Altıntaş, O. 1991. On a subclass of certain starlike functions with negative coefficient: Mathematica Japonica, 36: 489-495.

21. Altıntaş, O., Irmak, H. and Srivastava, H. M. 1995. Fractional calculus and certain starlike functions with negative coefficients: Computers and Mathematics with Applications, 30: 9-16.

22. Altıntaş, O., Özkan, Ö. And Srivistava, H. M. 2004. Neighbourhoods of a Certain Family of Multivalent Functions with Negative Coefficients: Computers and Mathematics with Applications, 47: 1667- 1672.

23. Porwal, S. 2014. An application of a Poisson distribution series on certain analytic functions: Journal of Complex Analysis, Article ID 984135, pp. 1-3.

24. Porwal, S. 2014. An application of a Poisson distribution series on certain analytic functions :Journal of Complex Analysis, Article ID 984135, pp. 1-3. 